\title{
Compressão de Sinais Elétricos Usando a Transformada de Wavelet ${ }^{1}$
}

\author{
M.A.Q. DUARTE 2 , L.C.O. de OLIVEIRA ${ }^{3}$, PPG Engenharia Elétrica, Faculdade \\ de Engenharia de Ilha Solteira, UNESP, 15385-000 Ilha Solteira, SP, Brasil. \\ F. VILLARREAL ${ }^{4}$, Depto. de Matemática, Faculdade de Engenharia de Ilha \\ Solteira, UNESP, 15385-000 Ilha Solteira, SP, Brasil. \\ L.A. DÍAZ ${ }^{5}$, Dirección de Ciencias, Capitolio de La Habana, 10.600, Havana, \\ Cuba.
}

Resumo. O objetivo deste trabalho é apresentar a transformada de wavelet como uma ferramenta eficaz no processamento de sinais provenientes de transdutores em sistemas de energia elétrica para análises na área de qualidade de energia elétrica. Propõe-se a aplicação da transformada de wavelet para compressão de sinais de forma que a quantidade de informações a respeito destes seja significativamente reduzida, através dos sinais transformados, no sentido de minimizar o espaço de memória utilizada para armazená-los. Neste trabalho, é enfocado particularmente o item de qualidade de energia associado à presença de harmônicos nos sinais de tensão e corrente. A partir da compressão de dados em sinais com alto conteúdo harmônico, discutem-se as potencialidades da transformada de wavelet no sentido de garantir uma precisão adequada no cálculo dos harmônicos em função da taxa de compressão utilizada, e conseqüentemente da memória economizada.

\section{Introdução}

Apesar dos esforços conjuntos desempenhados pelas concessionárias e consumidores, observa-se atualmente que os índices de distorção harmônica nas tensões e correntes têm aumentado, ultrapassando muitas vezes os níveis máximos admissíveis ou recomendados. Nestas condições, os prejuízos para os supridores e/ou para os consumidores poderão atingir proporções alarmantes e, somente com um controle consciente, efetivo e sistematizado, envolvendo as duas partes citadas, podem-se evitar ou reduzir conseqüências danosas e operações elétricas indevidas. Objetivando-se

\footnotetext{
${ }^{1}$ Trabalhos apresentados no CNMAC.

${ }^{2}$ marco@dee.feis.unesp.br

3 origa@dee.feis.unesp.br

${ }^{4}$ villa@fqm.feis.unesp.br

${ }^{5}$ lilliam@ceniai.inf.cu
} 
identificar e quantificar os níveis harmônicos nos sistemas elétricos, várias ações têm sido direcionadas no sentido de estabelecer limites adequados e metodologias de monitoramento e processamento dos sinais amostrados.

O Operador Nacional do Sistema (ONS), vem desenvolvendo ações em parceria com instituições de pesquisa no sentido de estabelecer limites para as distorções harmônicas, definir metodologia para medição e gerenciamento de um banco de dados global de todo o sistema elétrico brasileiro.

O protocolo de medições proposto, em sua essência, objetiva estabelecer uma metodologia a partir da qual os resultados parciais sejam tratados e culminem num valor de distorção harmônica que represente a situação operacional do ponto monitorado. Atualmente, os entendimentos encaminhados pelas entidades de pesquisa junto ao ONS, sugerem que o intervalo de janela de medição para cada amostra esteja entre $80 \mathrm{~ms}$ e $500 \mathrm{~ms}$. Sabendo-se também que a taxa de aquisição indicada é de, no mínimo $6 k H z$, ou seja, 100 ponto/ciclo, verifica-se que o total de pontos a serem armazenados por amostra pode variar aproximadamente entre 500 e 3000 [5]. Segundo o protocolo proposto, este volume de informações deverá produzir o primeiro resultado prático ao final de 5 segundos, definido como intervalo de curtíssima duração [2], onde nova amostra a ser armazenada será obtida calculandose a média quadrática das amostras anteriores. Para este cálculo o total de pontos armazenados pode chegar a 30000. Na seqüência, uma nova média quadrática deve ser calculada a partir daquelas obtidas nos intervalos de curtíssima duração. Esta nova média a ser obtida no chamado intervalo de curta duração, deve considerar 10 minutos, totalizando cerca de 600000 pontos.

Fica então evidente, que na medida em que o protocolo de medição vem sendo cumprido, até que se obtenha um resultado representativo ao longo de uma semana, existirá sempre um grande número de informações a serem processadas e armazenadas. Por outro lado, almejando também dispor de uma base de dados do sistema elétrico brasileiro, deve-se reconhecer o grande volume de informações a serem transmitidas e armazenadas na medida em que todas as concessionárias deverão contribuir para este objetivo. Os aspectos quantitativos e qualitativos envolvidos com as definições dos locais e número de pontos a serem monitorados, são difíceis de se estabelecer. Considerando, entretanto, as normas internacionais, as especificidades de cada empresa supridora, os recursos humanos e os materiais necessários aos trabalhos para cada um dos diferentes tipos de consumidores, sugere-se uma porcentagem mínima de pontos a serem monitorados. A título de ilustração, considerando-se uma cidade com 100.000 consumidores residenciais e/ou comerciais em que cada transformador de distribuição alimente 50 consumidores, para tal sistema de distribuição haveria um total de 2.000 transformadores. Nos termos propostos pelo ONS, no mínimo 1\% deste total, ou seja, 20 unidades devem ser monitoradas anualmente, aplicando-se para cada uma delas o protocolo de medição proposto. Desta forma, para se obter os resultados almejados, busca-se a utilização de ferramentas que venham contribuir para uma manipulação de dados, otimizando a utilização da memória e garantindo a integridade dos resultados. Neste sentido, objetivando a otimização da memória, este trabalho apresenta a aplicação da transformada de wavelet como instrumento de apoio para compactação de da- 
dos. O método utilizado é praticamente o mesmo apresentado em [3], porém, neste caso, enfoca-se a precisão no cálculo das componentes harmônicas obtidas através da transformada de Fourier (FFT) e a distorção harmônica total (DHT).

\section{A transformada de Wavelet}

Por se tratar de uma técnica de janelamento instantânea, a transformada de wavelet é útil no processamento de sinais não estácionários[6].

A transformada de wavelet é, basicamente, uma operação de filtragem [1, 4], através de um filtro passa baixas $h[n]$ e um filtro passa banda $g[n]$, que decompõem o sinal $c_{0}[n]$ em $c_{1}[n]$ e $d_{1}[n]$, respectivamente. Como os sinais decompostos estão localizados em duas faixas diferentes, temos uma decomposição em duas faixas. $\mathrm{O}$ sinal $c_{1}[n]$ é obtido através da convolução de $c_{0}[n] \operatorname{com} h[n]$ mais uma operação de subamostragem de ordem $2, d_{1}[n]$ também é obtido por convolução, mas de $c_{0}[n]$ com $g[n]$ e também uma operação de subamostragem de ordem 2, a Figura 1 ilustra este processo. Assim, $c_{1}[n]$ contém as componentes de baixas freqüências e $d_{1}[n]$ contém as componentes de altas freqüências de $c_{0}[n] . \quad c_{1}[n]$ contém os chamados coeficientes de aproximação e $d_{1}[n]$, os coeficientes de detalhes. As equações (2.1) e (2.2), apresentadas a seguir, expressam as relações entre $c_{0}[n], c_{1}[n]$ e $d_{1}[n]$.

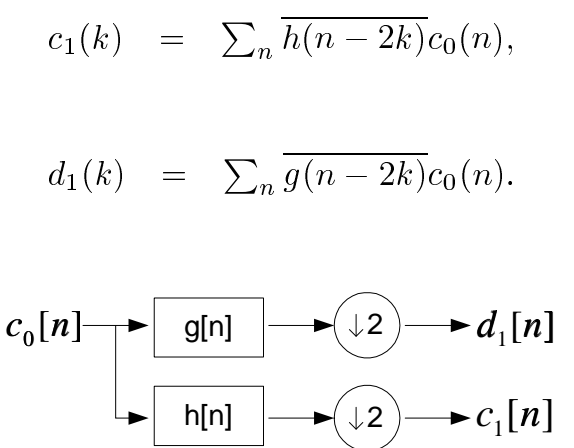

Figura 1: Decomposição em duas faixas

Depois de decomposto pela transformada de wavelet, fazem-se as operações desejadas e o sinal original pode ser reconstruído pela trasformada de wavelet inversa $[1,4]$. Neste trabalho a operação realizada é a compressão. A Figura 2 mostra este processo de reconstrução. Primeiro insere-se um zero entre cada elemento dos sinais $c_{1}[n]$ e $d_{1}[n]$ (sobreamostragem de ordem 2); depois fazem-se as convoluções de $c_{1}[n]$ com $h[n]$ e $d_{1}[n]$ com $g[n]$. O sinal reconstruído é, então, a soma destes dois sinais convoluídos, conforme expresso na equação (2.3).

$$
c_{0}(n)=\sum_{k} h(n-2 k) c_{1}(k)+\sum_{k} g(n-2 k) d_{1}(k)
$$




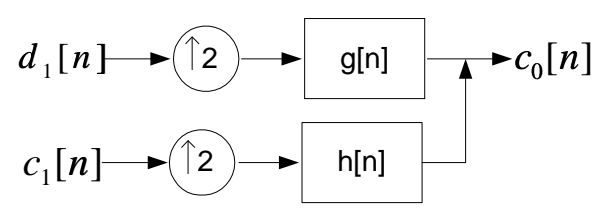

Figura 2: Reconstrução em duas faixas

A operação de filtragem em duas faixas pode ser estendida para uma decomposição em $k$ faixas, sendo $k$ o número de faixas de freqüências nas quais os sinais decompostos estão localizados. As Figuras 3 e 4 ilustram, respectivamente, os processos de decomposição e reconstrução de um sinal em três faixas. O sinal $c_{0}[n]$ é decomposto em componentes de baixas e altas freqüências, $c_{1}[n]$ e $d_{1}[n]$; em seguida, $c_{1}[n]$ é novamente decomposto em componentes de baixas e altas freqüências, $c_{2}[n]$ e $d_{2}[n]$. Este processo é repetido conforme o número de faixas em que se quer decompor o sinal. No método proposto, apresentado a seguir, para um sinal com $m$ pontos amostrais, a decomposição é feita em $k=\log _{2} m$ faixas, que é o máximo de faixas de decomposição que podem ser usadas [4].

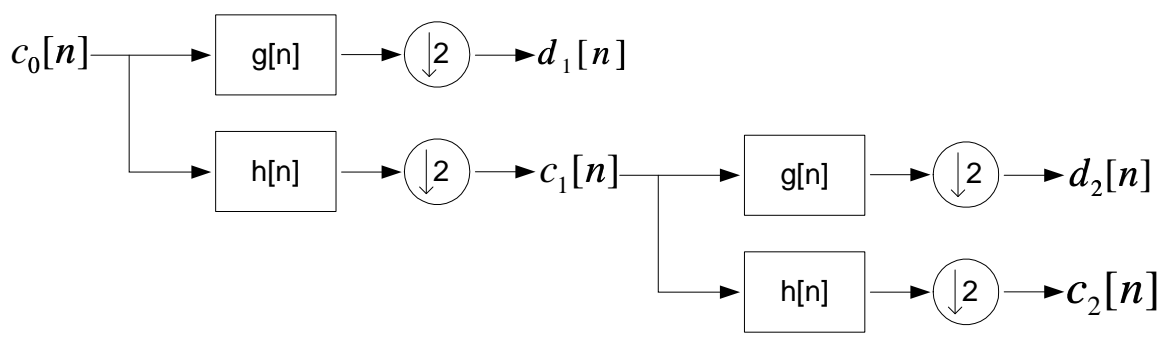

Figura 3: Decomposição em três faixas

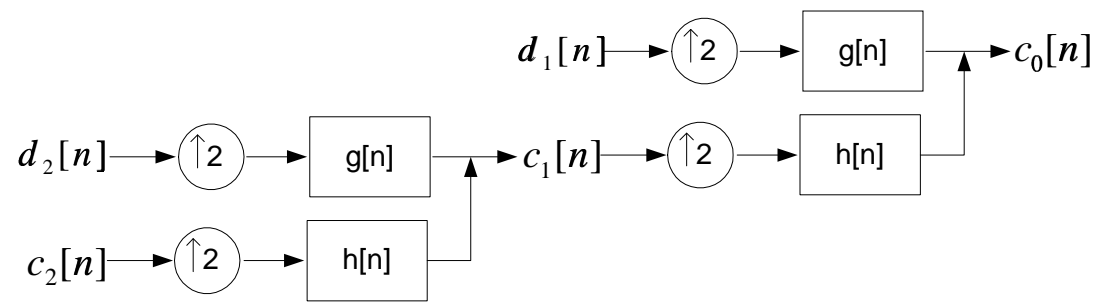

Figura 4: Reconstrução em três faixas

\section{O método proposto}

Com o objetivo de manter os valores numéricos dos diferentes sinais amostrados numa mesma ordem de grandeza, os dados reais, amostrados através de um sistema 
de aquisição de dados, são armazenados em p.u. (por unidade), tomando-se por base o valor da maior amostra, conforme a equação (3.1).

$$
c_{0}(n)=\frac{C_{0}(n)}{C_{0 \max }} .
$$

Sendo $C_{0}(n)$, o valor real da n-ésima amostra e, $C_{0 \text { max }}$, o maior valor absoluto entre as $n$ amostras.

A estrutura do método proposto é apresentada na Figura 5. Conforme a figura ilustra, primeiro aplica-se a transformada de wavelet no sinal original. Depois, fazse a compressão no sinal transformado, eliminando todas as componentes que estão abaixo do limite especificado, atribuindo para estas o valor zero. Após a compressão, o sinal é reconstruído a partir do novo sinal transformado.

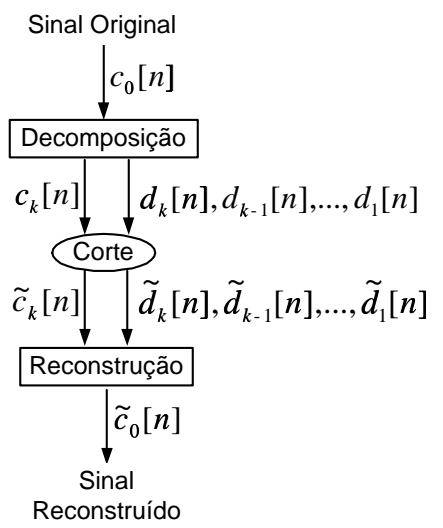

Figura 5: Estrutura do método proposto

\subsection{O Corte}

Na decomposição dos sinais elétricos utilizados, $c_{k}[n]$ e $d_{k}[n], d_{k-1}[n], \ldots, d_{1}[n]$ contêm vários elementos que estão bem próximos de zero, assim a eliminação destes valores não causaria muitos danos na reconstrução do sinal original. As equações (3.2) e (3.3) abaixo definidas são utilizadas para a compressão em $c_{k}[n]$ e $d_{k}[n]$, $d_{k-1}[n], \ldots, d_{1}[n]:$

$$
\begin{aligned}
& \widetilde{c}_{i}[n]=\left\{\begin{array}{rll}
c_{i}[n], & \text { se } & \left|c_{i}[n]\right| \geq \epsilon \\
0, & \text { se } & \left|c_{i}[n]\right|<\epsilon
\end{array}, i=1, \ldots, k,\right. \\
& \widetilde{d}_{i}[n]=\left\{\begin{array}{rll}
d_{i}[n], & \text { se } & \left|d_{i}[n]\right| \geq \epsilon \\
0, & \text { se } & \left|d_{i}[n]\right|<\epsilon
\end{array}, i=1, \ldots, k .\right.
\end{aligned}
$$

O valor do limite $\epsilon$ é calculado em p.u. (por unidade), tomando-se como base o maior valor obtido entre as $n$ amostras, conforme a equação (3.4). Naturalmente, quanto maior for o valor de $\epsilon$, maior será a taxa de compressão. 


$$
\epsilon=\frac{C_{0 \min }}{C_{0 \max }},
$$

sendo $C_{0 \min }$ o menor valor a ser mantido no sinal amostrado.

\section{Testes e resultados}

Para os sinais analisados, a transformada de wavelet foi implementada usando a wavelet de Haar; levou-se em consideração o erro percentual na relação sinal original/sinal comprimido comparando as respectivas componentes harmônicas dos dois sinais, bem como a distorção harmônica total (DHT), calculada conforme a equação (4.1).

Para testes com o método proposto, foram usados dois sinais com 512 pontos amostrais cada. O primeiro sinal (A) contém as seguintes componentes harmônicas: terceira, quinta, décima primeira e vigésima terceira. Na Tabela 1, são apresentados os resultados obtidos após compressão deste sinal, no cálculo das harmônicas e da DHT. O sinal original é ilustrado na Figura 6 e, na Figura 7, apresentam-se os espectros harmônicos obtidos para o sinal original e para o sinal comprimido em 43,94\%. A Figura 8 ilustra graficamente o comportamento do erro percentual cometido no cálculo das diferentes componentes harmônicas e da DHT a partir dos sinais comprimidos com relação ao sinal A. O segundo sinal (B) contém as seguintes componentes harmônicas: terceira, quinta, décima e vigésima. Na Tabela 2, são apresentados os resultados obtidos após a compressão deste sinal, no cálculo das harmônicas e da DHT. O sinal original é ilustrado na Figura 9 e, na Figura 10, apresentam-se os espectros harmônicos obtidos para o sinal original e para o sinal comprimido em 44,53\%. A Figura 11 ilustra graficamente o comportamento do erro percentual cometido no cálculo das diferentes componentes harmônicas e da DHT a partir dos sinais comprimidos com relação ao sinal B.

$$
D H T=\sqrt{\sum_{j=1}^{n} I_{j}^{2} / I_{1}^{2}} .
$$

Sendo $I_{j}$ o valor eficaz da j-ésima componente harmônica do sinal obtida na FFT e $I_{1}$ o valor eficaz da componete harmônica fundamental obtida na FFT.

\begin{tabular}{|c|c|c|c|c|c|c|c|c|}
\hline \multirow[b]{2}{*}{ corte } & \multirow{2}{*}{$\begin{array}{c}\text { compressão } \\
\left(n^{\mathbf{o}} \text { de pontos }\right. \\
\text { eliminados })\end{array}$} & \multirow{2}{*}{$\operatorname{taxa}(\%)$} & \multicolumn{6}{|c|}{ erro (\%) } \\
\hline & & & fund & $3^{\underline{a}}$ & $5^{\underline{\mathbf{a}}}$ & $11^{\mathbf{a}}$ & $23^{\underline{a}}$ & DHT \\
\hline 0.100 & 131 & 25.60 & 1.09 & 1.68 & 0.10 & 0.09 & 1.33 & 1.32 \\
\hline 0.125 & 144 & 28.12 & 1.43 & 1.50 & 0.17 & 0.06 & 1.09 & 1.31 \\
\hline 0.150 & 170 & 33.20 & 3.58 & 2.06 & 0.43 & 1.34 & 0.84 & 3.11 \\
\hline 0.175 & 204 & 39.84 & 3.76 & 1.89 & 2.11 & 0.48 & 1.13 & 2.60 \\
\hline 0.200 & 225 & 43.94 & 3.94 & 0.89 & 1.06 & 2.07 & 2.21 & 2.30 \\
\hline 0.225 & 249 & 48.63 & 4.04 & 0.38 & 2.19 & 1.22 & 5.19 & 1.52 \\
\hline 0.250 & 263 & 51.36 & 6.44 & 3.49 & 4.51 & 1.56 & 5.30 & 2.12 \\
\hline
\end{tabular}

Tabela 1: Resultado da compressão - sinal A 


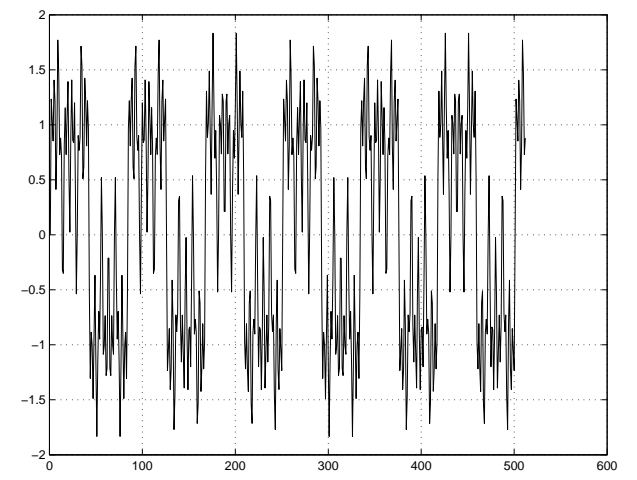

Figura 6: Sinal A

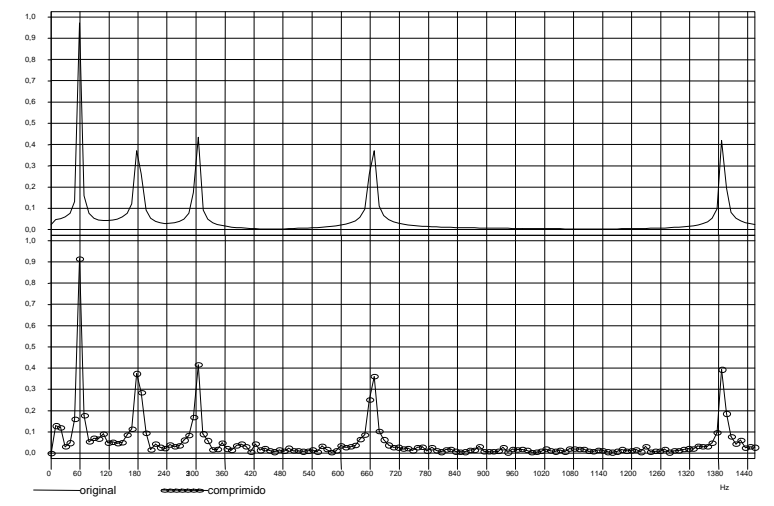

Figura 7: Espectro harmônico do sinal original e do sinal comprimido em 43,94\%

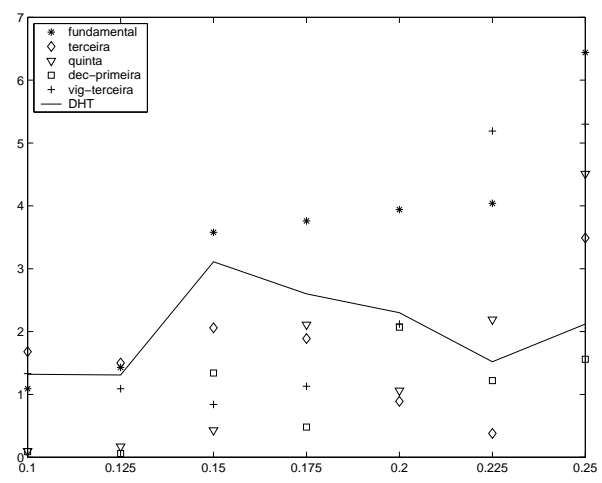

Figura 8: Erro no cálculo das harmônicas e da DHT em função da taxa de compressão do sinal A 
Tabela 2: Resultado da compressão - sinal B

\begin{tabular}{|c|c|c|c|c|c|c|c|c|}
\hline \multirow{2}{*}{ corte } & \multirow{2}{*}{$\begin{array}{c}\text { compressão } \\
(n \underline{\mathbf{0}} \text { de pontos } \\
\text { eliminados) }\end{array}$} & \multirow{2}{*}{ taxa (\%) } & & \multicolumn{6}{|c|}{ erro (\%) } \\
\cline { 4 - 9 } & 129 & 25.20 & 0.77 & 0.87 & 0.03 & 1.49 & 0.43 & 0.48 \\
\hline 0.100 & 158 & 30.86 & 0.74 & 0.62 & 0.14 & 1.85 & 1.14 & 0.39 \\
\hline 0.125 & 182 & 35.54 & 2.82 & 1.77 & 0.71 & 2.59 & 0.76 & 1.94 \\
\hline 0.150 & 200 & 39.06 & 3.72 & 2.94 & 1.21 & 2.90 & 0.36 & 2.80 \\
\hline 0.175 & 228 & 44.53 & 3.47 & 2.24 & 1.47 & 2.63 & 2.69 & 1.96 \\
\hline 0.200 & 249 & 48.63 & 3.73 & 1.76 & 1.13 & 3.82 & 3.88 & 1.69 \\
\hline 0.225 & 275 & 53.71 & 6.01 & 0.69 & 3.89 & 6.85 & 3.40 & 0.99 \\
\hline 0.250 & & & & & & & & \\
\hline
\end{tabular}

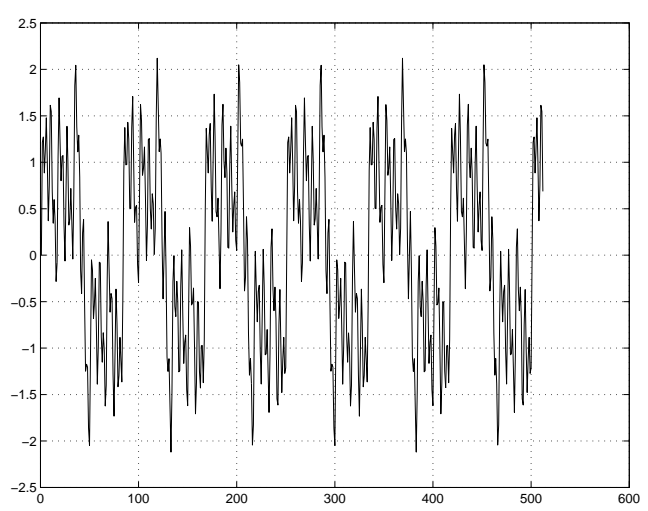

Figura 9: Sinal B

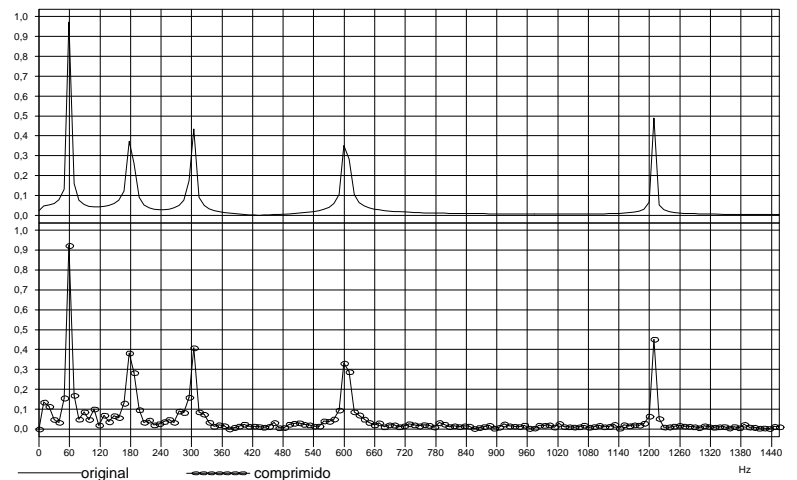

Figura 10: Espectro harmônico do sinal original e do sinal comprimido em 44,53\% 


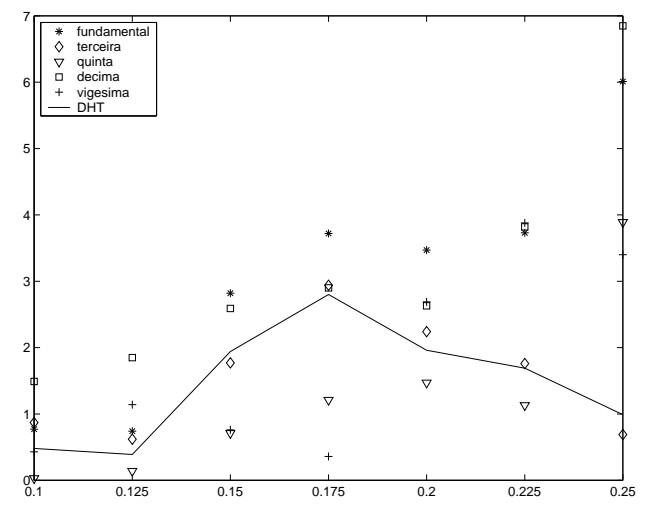

Figura 11: Erro no cálculo das harmônicas e da DHT em função da taxa de compressão do sinal B

\section{Conclusões}

Neste trabalho, com o objetivo de armazenamento de sinais elétricos, propôs-se um método de compressão utilizando-se da transformada de wavelet com o objetivo de reduzir o espaço de memória utilizado para armazenamento dos mesmos. Para tanto, utilizou-se a wavelet de Haar, obtendo-se bons resultados. Os resultados obtidos para o cálculo das componentes harmônicas e da DHT podem ser considerados satisfatórios para taxas de compressão de até $45 \%$, pois, para todos os casos simulados com taxa inferior a $45 \%$, o erro percentual cometido permaneceu abaixo de $5 \%$ para todas as ordens harmônicas calculadas e para a DHT. Sob o ponto de vista da economia de memória os resultados são relevantes, uma vez que o número de pontos a serem armazenados após a compressão, mantendo-se o erro dentro dos limites de tolerância do aparelho de medida [5], é praticamentete a metade do total de pontos amostrados no sinal original. Desta forma, a transformada de wavelet mostrouse adequada para a aplicação proposta neste trabalho, sendo que outros tipos de funções wavelets podem ainda ser testadas em busca de melhores resultados.

Abstract. The purpose of this work is to present the wavelet transform as an efficient tool for processing signals from transducers in power electric systems for analysis in the power electric quality. It is proposed the wavelet transform application to signals compression in a way that the information amount in terms of these signals would be expensively reduced, trough the transformed signals, to minimize the space of memory utilized to save them. In this work, it is particularly emphasized the item power quality associated to the harmonics presence in the signals of voltage and current. From signals data compression with high harmonics content, it is discussed the wavelet transform potentiality to assure an appropriate precision in the harmonics computation in terms of the used compression rate, and consequently of the saved memory. 


\section{Referências}

[1] I. Daubechies, "Ten Lectures on Wavelets", SIAM, 1992.

[2] N.S.D. Brito, "Teoria wavelet e suas aplicações em sistemas de energia elétrica", Dissertação de Mestrado, Unicamp, Campinas, SP, 1996.

[3] H. Cheng-Tao, S. Huang e C. Huang, Data reduction of power disturbances - A wavelet transform approach, Eletric Power Systems Research, 47 (1998), 79-86.

[4] Y. Nievergelt, "Wavelets made easy", Birkhäuser, Boston, 1999.

[5] ONS, Relatórios preliminares do Operador Nacional do Sistema, Rio de Janeiro, 2002.

[6] O. Rioul e M. Vetterli, Wavelets and Signal Processing, IEEE Signal Processing Magazine, 8, No. 4 (1991), 14-38. 